\title{
THE WEIL REPRESENTATION AND HECKE OPERATORS FOR VECTOR VALUED MODULAR FORMS
}

\author{
JAN HENDRIK BRUINIER AND OLIVER STEIN
}

\begin{abstract}
We define Hecke operators on vector valued modular forms transforming with the Weil representation associated to a discriminant form. We describe the properties of the corresponding algebra of Hecke operators and study the action on modular forms.
\end{abstract}

\section{INTRODUCTION}

Hecke operators are a fundamental tool in the study of modular forms. They can be used to obtain information on the arithmetic nature of the Fourier coefficients. They are vital for the definition of $L$-functions associated to modular forms and for understanding their properties. The theory of Hecke operators is well developed for scalar valued modular forms [Sh1].

In many recent works, vector valued modular forms associated to the Weil representation play an important role, see e.g. [Bo1, [Bo2, [Br], $\mathrm{McG}$, [Sch. For instance, Borcherds uses them to provide a elegant description of the Fourier expansion of various theta liftings. The purpose of the present paper is to develop the foundations of a Hecke theory for such vector valued modular forms. The results can be used to associate an $L$-function to a vector valued modular form, essentially the standard $L$-function.

We now describe the content of this paper in more detail. Let $L$ be a non-degenerate even lattice of type $\left(b^{+}, b^{-}\right)$and level $N$. The modulo 1 reduction of the quadratic form on the dual lattice $L^{\prime}$ defines a $\mathbb{Q} / \mathbb{Z}$-valued quadratic form on the discriminant group $A=L^{\prime} / L$. To simplify the exposition, we assume throughout the introduction that the signature $\operatorname{sig}(L)=b^{+}-b^{-}$of $L$ is even. In the body of the paper, both, odd and even signature is treated.

The Weil representation associated to the discriminant form $A$ is a unitary representation of $\Gamma=\mathrm{SL}_{2}(\mathbb{Z})$ on the group ring $\mathbb{C}[A]$,

$$
\rho_{A}: \Gamma \longrightarrow \mathrm{U}(\mathbb{C}([A])),
$$

defined by (2.3), (2.4). It factors through the finite quotient

$$
S(N):=\mathrm{SL}_{2}(\mathbb{Z} / N \mathbb{Z}) \cong \Gamma / \Gamma(N)
$$

Date: October 27, 2018.

2000 Mathematics Subject Classification. 11F27, 11F25. 
Let $k \in \mathbb{Z}$. A holomorphic function $f: \mathbb{H} \rightarrow \mathbb{C}[A]$ is called a modular form of weight $k$ and type $\rho_{A}$ for the group $\Gamma$, if

$$
f(M \tau)=(c \tau+d)^{k} \rho_{A}(M) f(\tau)
$$

for all $M=\left(\begin{array}{ll}a & b \\ c & d\end{array}\right) \in \Gamma$, and $f$ is holomorphic at the cusp $\infty$. We denote the vector space of such holomorphic modular forms by $M_{k, A}$, and write $S_{k, A}$ for the subspace of cusp forms.

In order to define Hecke operators on vector valued modular forms of type $\rho_{A}$, we need to extend the representation $\rho_{A}$ to a sufficiently large subgroup of $\mathrm{GL}_{2}^{+}(\mathbb{Q})$. A natural starting point is to try to extend $\rho_{A}$, viewed as a representation of $S(N)$, to a representation of $G(N):=\mathrm{GL}_{2}(\mathbb{Z} / N \mathbb{Z})$. However, it was observed by E. Freitag that such an extension does not exist in general, see Example 3.1 .

Here we consider the subgroup

$$
\{M \in G(N) ; \operatorname{det}(M) \equiv \square(\bmod N)\}
$$

of matrices whose determinant is a square modulo $N$. It has the extension

$$
Q(N)=\left\{(M, r) \in G(N) \times U(N) ; \quad \operatorname{det}(M) \equiv r^{2} \quad(\bmod N)\right\},
$$

where $U(N)$ denotes the unit group of $\mathbb{Z} / N \mathbb{Z}$. The group $Q(N)$ is isomorphic to $S(N) \times$ $U(N)$. Consequently, we may extend the Weil representation to $Q(N)$ by taking the tensor product of $\rho_{A}$ on $S(N)$ and a suitable character on $U(N)$, see Proposition 3.3.

If $M$ is an element of $G(N)$ whose determinant is a square modulo $N$, and $r, r^{\prime} \in U(N)$ with $\operatorname{det}(M) \equiv r^{2} \equiv r^{\prime 2}(\bmod N)$, then $(M, r)$ and $\left(M, r^{\prime}\right)$ both belong to $Q(N)$. We prove that the action of $\rho_{A}(M, r)$ and $\rho_{A}\left(M, r^{\prime}\right)$ on $\mathbb{C}[A]$ differ only by the action of an element of the orthogonal group $\mathrm{O}(A)$, see Proposition 3.5.

This extension of the Weil representation can be used to define a Hecke operator $T(M, r)$ on $M_{k, A}$ for every pair $(M, r)$, where $M \in \mathrm{M}_{2}(\mathbb{Z})$ and $r \in U(N)$ with $\operatorname{det}(M) \equiv r^{2}$ $(\bmod N)$. We compute the action of these operators on the Fourier expansion of a modular form (see Section 4.1). They generalize the classical Hecke operators on scalar valued modular forms and Jacobi forms (see e.g. Remark 4.4 and Remark 4.11).

In particular, for every positive integer $m$ coprime to $N$ we obtain a Hecke operator

$$
T\left(m^{2}\right)^{*}:=T\left(\left(\begin{array}{cc}
m^{2} & 0 \\
0 & 1
\end{array}\right), m\right)
$$

on $M_{k, A}$. These operators generate a commutative subalgebra of $\operatorname{End}\left(M_{k, A}\right)$, which is actually already generated by the $T\left(p^{2}\right)^{*}$ for $p$ prime and coprime to $N$. The operators $T\left(m^{2}\right)^{*}$ take cusp forms to cusp forms and are self-adjoint with respect to the Petersson scalar product (see Theorem 4.12). In particular, $S_{k, A}$ has a basis of simultaneous eigenforms of all $T\left(m^{2}\right)^{*}$ with $(m, N)=1$.

In Section $\left[5\right.$ we extend the definition of the Hecke operators $T\left(m^{2}\right)^{*}$ to all positive integers $m$, not necessarily coprime to $N$. This is done by defining the right-action on $\mathbb{C}[A]$ of a matrix $\alpha=\left(\begin{array}{cc}m^{2} & 0 \\ 0 & 1\end{array}\right)$ by the same formula as in the case where $m$ is coprime to $N$. Notice, that the corresponding linear map

$$
\mathbb{C}[A] \longrightarrow \mathbb{C}[A],\left.\quad \mathfrak{e}_{\lambda} \mapsto \mathfrak{e}_{\lambda}\right|_{A} \alpha=\mathfrak{e}_{m \lambda}
$$


is neither surjective nor injective in general. However, it still can be used to obtain an "action" of the double coset $\Gamma \alpha \Gamma$, see Proposition 5.1 and Lemma 5.2. This suffices to define a corresponding Hecke operator $T\left(m^{2}\right)^{*}$ on $M_{k, A}$, which is consistent with our earlier definition when $m$ is coprime to $N$.

For any positive integer $m$, the Hecke operator $T\left(m^{2}\right)^{*}$ is self adjoint with respect to the Petersson scalar product. Moreover, if $m$ and $n$ are coprime, then

$$
T\left(m^{2}\right)^{*} T\left(n^{2}\right)^{*}=T\left(m^{2} n^{2}\right)^{*},
$$

see Theorem 5.6. Observe that for a prime $p$ dividing $N$ the local Hecke algebra, that is, the subalgebra of $\operatorname{End}\left(M_{k, A}\right)$ generated by the $T\left(p^{2 \nu}\right)^{*}$, is considerably more complicated than in the case where $p$ is coprime to $N$. For instance, it is commutative if $p$ is coprime to $N$, but in general non-commutative if $p$ divides $N$.

Let $S$ be a finite set of primes and let $N_{S}$ be the product of the primes in $S$. Let $f \in S_{k, A}$ be a common eigenform of all $T\left(m^{2}\right)^{*}$ with $\left(m, N_{S}\right)=1$, so

$$
\left.f\right|_{k, A} T\left(m^{2}\right)^{*}=\lambda_{m}(f) f .
$$

We can use the above results to define an $L$-function associated to $f$ by putting

$$
L^{S}(s, f)=\sum_{\substack{m \geq 1 \\\left(m, N_{S}\right)=1}} \lambda_{m}(f) m^{-s} .
$$

It is easily seen that $L^{S}(s, f)$ converges for $\Re(s)$ sufficiently large. By Theorem 5.6, this $L$-function has an Euler product expansion. According to [Bö], it should be viewed as the standard $L$-function of $f$. It would be interesting to study the analytic properties of $L^{S}(s, f)$ in more detail. This could possibly be done by using a variant of the doubling method (see [B̈̈], Ga], [PSR]) involving a Siegel Eisenstein series of genus 2 associated to the Weil representation of $\operatorname{Sp}(2, \mathbb{Z})$ on $\mathbb{C}\left[A^{2}\right]$.

Moreover, it would be very interesting to develop a theory of new forms for the space $M_{k, A}$. One could try to associate an irreducible automorphic representation to a vector valued new form and study the properties of the resulting map.

If the signature of $L$ is odd, one can carry over the above results. However, one has to work with the metaplectic cover of $\Gamma$ and with similar $\{ \pm 1\}$-extensions of $S(N), G(N)$, and $Q(N)$. In this case $M_{k, A}$ vanishes unless $k$ is half-integral. Following the argument of Shimura [Sh2], we show that the Hecke operator $T(M, r)$ vanishes identically unless $\operatorname{det}(M)$ is the square of a rational number, see Proposition 4.9. The computation of the action of the Hecke operators on modular forms is more involved than in the even signature (i.e. integral weight) case, see Theorem 4.10.

We thank E. Freitag for many valuable discussions on this paper. Moreover, we thank J. Funke for several useful comments.

\section{Discriminant Forms And the Weil RePresentation}

Here we briefly summarize some facts on lattices, discriminant forms, and the Weil representation. See also [Bo1], [Bo2], [Br]. 
Let $L$ be a non-degenerate even lattice of type $\left(b^{+}, b^{-}\right)$. We denote the bilinear form on $L$ by $(\cdot, \cdot)$ and the associated quadratic form by $x \mapsto \frac{1}{2} x^{2}=\frac{1}{2}(x, x)$. We let $\operatorname{sig}(L)=b^{+}-b^{-}$ be the signature of $L$. We write $L^{\prime}$ for the dual lattice of $L$, and denote by $N$ the level of $L$, that is, the smallest positive integer such that $\frac{N}{2} x^{2} \in \mathbb{Z}$ for all $x \in L^{\prime}$. The finite abelian group $L^{\prime} / L$ is called the discriminant group of $L$. Its order is equal to the absolute value of the Gram determinant of $L$.

Recall that a discriminant form is a finite abelian group $A$ together with a $\mathbb{Q} / \mathbb{Z}$-valued non-degenerate quadratic form $x \mapsto \frac{1}{2} x^{2}$, for $x \in A$ (see [Ni]). If $L$ is a non-degenerate even lattice then $L^{\prime} / L$ is a discriminant form where the quadratic form is given by the mod 1 reduction of the quadratic form on $L^{\prime}$. Conversely, every discriminant form can be obtained in this way. The quadratic form on $L^{\prime} / L$ determines the signature of $L$ modulo 8 by Milgram's formula (see [MH] Appendix 4):

$$
\sum_{\lambda \in L^{\prime} / L} e\left(\lambda^{2} / 2\right)=\sqrt{\left|L^{\prime} / L\right|} e(\operatorname{sig}(L) / 8) .
$$

Here and throughout we abbreviate $e(z)=e^{2 \pi i z}$ for $z \in \mathbb{C}$. We define the signature $\operatorname{sig}(A) \in \mathbb{Z} / 8 \mathbb{Z}$ of a discriminant form $A$ to be the signature of any even lattice with that discriminant form.

If $A$ is a discriminant form, then we write $A^{n}$ for the subgroup of elements that are $n$-th powers of elements of $A$. Moreover, we write $A_{n}$ for the subgroup of elements of $A$ whose order divides $n$. We have an exact sequence

$$
0 \longrightarrow A_{n} \longrightarrow A \longrightarrow A^{n} \longrightarrow 0
$$

and $A^{n}$ is the orthogonal complement of $A_{n}$.

Let $\mathbb{H}=\{\tau \in \mathbb{C} ; \Im(\tau)>0\}$ by the complex upper half plane. We write $\widetilde{G L}_{2}^{+}(\mathbb{R})$ for the metaplectic two-fold cover of $\mathrm{GL}_{2}^{+}(\mathbb{R})$. The elements of this group are pairs $(M, \phi(\tau))$ where $M=\left(\begin{array}{ll}a & b \\ c & d\end{array}\right) \in \mathrm{GL}_{2}^{+}(\mathbb{R})$ and $\phi: \mathbb{H} \rightarrow \mathbb{C}$ is a holomorphic function with $\phi(\tau)^{2}=c \tau+d$. The multiplication is defined by

$$
(M, \phi(\tau))\left(M^{\prime}, \phi^{\prime}(\tau)\right)=\left(M M^{\prime}, \phi\left(M^{\prime} \tau\right) \phi^{\prime}(\tau)\right) .
$$

For $g=(M, \phi) \in{\widetilde{\mathrm{GL}_{2}}}_{2}^{+}(\mathbb{R})$, we put $\operatorname{det}(g)=\operatorname{det}(M)$. Moreover, if $G$ is a subset of $\mathrm{GL}_{2}^{+}(\mathbb{R})$, we write $\tilde{G}$ for its inverse image under the covering map. Throughout we write $\Gamma=\mathrm{SL}_{2}(\mathbb{Z})$ for the full modular group. It is well known that the integral metaplectic group $\tilde{\Gamma}$ is generated by $T=\left(\left(\begin{array}{ll}1 & 1 \\ 0 & 1\end{array}\right), 1\right)$, and $S=\left(\left(\begin{array}{cc}0 & -1 \\ 1 & 0\end{array}\right), \sqrt{\tau}\right)$. One has the relations $S^{2}=(S T)^{3}=Z$, where $Z=\left(\left(\begin{array}{cc}-1 & 0 \\ 0 & -1\end{array}\right), i\right)$ is the standard generator of the center of $\tilde{\Gamma}$.

We now recall the Weil representation associated with a discriminant form $A$ (see also [Bo1], Bo2]). It is a representation of $\tilde{\Gamma}$ on the group algebra $\mathbb{C}[A]$. We denote the standard basis elements of $\mathbb{C}[A]$ by $\mathfrak{e}_{\lambda}, \lambda \in A$, and write $\langle\cdot, \cdot\rangle$ for the standard scalar product (antilinear in the second entry) such that $\left\langle\mathfrak{e}_{\lambda}, \mathfrak{e}_{\mu}\right\rangle=\delta_{\lambda, \mu}$. The Weil representation $\rho_{A}$ associated with the discriminant form $A$ is the unitary representation of $\tilde{\Gamma}$ on the group 
algebra $\mathbb{C}[A]$ defined by

$$
\begin{aligned}
\rho_{A}(T)\left(\mathfrak{e}_{\lambda}\right) & =e\left(\lambda^{2} / 2\right) \mathfrak{e}_{\lambda}, \\
\rho_{A}(S)\left(\mathfrak{e}_{\lambda}\right) & =\frac{e(-\operatorname{sig}(A) / 8)}{\sqrt{|A|}} \sum_{\mu \in A} e(-(\lambda, \mu)) \mathfrak{e}_{\mu} .
\end{aligned}
$$

Note that

$$
\rho_{A}(Z)\left(\mathfrak{e}_{\lambda}\right)=e(-\operatorname{sig}(A) / 4) \mathfrak{e}_{-\lambda} .
$$

The orthogonal group $\mathrm{O}(A)$ also acts on $\mathbb{C}[A]$ by

$$
\rho_{A}(h)\left(\mathfrak{e}_{\lambda}\right)=\mathfrak{e}_{h^{-1} \lambda}
$$

for $h \in \mathrm{O}(A)$, and the actions of $\tilde{\Gamma}$ and $\mathrm{O}(A)$ commute.

If the signature of $A$ is even, then (2.5) implies that $Z^{2}$ acts trivially. Hence, the Weil representation factors through $\Gamma$. Moreover, it is trivial on the principal congruence subgroup $\Gamma(N)$, where $N$ is the level of $A$, i.e., the level of any even lattice $L$ with $L^{\prime} / L=A$ (see e.g. [Eb], Chapter 3, Theorem 3.2). Therefore, $\rho_{A}$ factors through the finite group

$$
S(N):=\mathrm{SL}_{2}(\mathbb{Z} / N \mathbb{Z}) \cong \Gamma / \Gamma(N) .
$$

If the signature of $A$ is odd, we notice that the level of $A$ must be divisible by 4 . This follows from the oddity formula ([CS] p. $383(30)$ ) which implies that $A$ contains odd 2-adic Jordan components. On $\Gamma(4)$ the metaplectic cover has the section

$$
s: \Gamma(4) \longrightarrow \tilde{\Gamma}(4), \quad\left(\begin{array}{ll}
a & b \\
c & d
\end{array}\right) \mapsto\left(\left(\begin{array}{ll}
a & b \\
c & d
\end{array}\right),\left(\frac{c}{d}\right) \sqrt{c \tau+d}\right)
$$

given by the theta multiplier system. Here $\sqrt{\cdot}$ denotes the principal branch of the holomorphic square root. The same argument as at the end of the proof of Theorem 5.4 in Bo2 implies that $\rho_{A}$ is trivial on $s(\Gamma(N))$ and factors through the central extension of $S(N)$ by $\{ \pm 1\}$ given by

$$
S_{1}(N):=\tilde{\Gamma} / s(\Gamma(N)) .
$$

We will also need the action of $\rho_{A}$ on diagonal matrices in $S(N)$. Following [McG], for integers $a, d$ coprime to $N$ such that $a d \equiv 1(\bmod N)$, we put

$$
R_{d}:=S T^{d} S^{-1} T^{a} S T^{d} .
$$

It is easily checked that $R_{d}=(M, \phi)$ where $M \equiv\left(\begin{array}{ll}a & 0 \\ 0 & d\end{array}\right)(\bmod N)$.

Lemma 2.1. (See [McG] Lemma 4.6.) For a,d as above we have

$$
\rho_{A}\left(R_{d}\right) \mathfrak{e}_{\lambda}=\frac{g_{d}(A)}{g(A)} \mathfrak{e}_{d \lambda} .
$$

Here $g_{d}(A)$ denotes the Gauss sum

$$
g_{d}(A)=\sum_{\lambda \in A} e\left(d \lambda^{2} / 2\right)
$$


and $g(A)=g_{1}(A)$.

Notice that by Milgram's formula we have $g(A)=\sqrt{|A|} e(\operatorname{sig}(A) / 8)$. Moreover, one easily checks that $\left|g_{d}(A)\right|=\sqrt{|A|}$. If $r \in \mathbb{Z}$ is coprime to $N$, then we have $g_{d r^{2}}(A)=g_{d}(A)$. In particular, $g_{d}(A)=g_{a}(A)$. Finally, Lemma 2.1 and the fact that $\rho_{A}$ is a representation imply the relation

$$
\frac{g_{d r}(A) g(A)}{g_{d}(A) g_{r}(A)}= \begin{cases}1, & \text { if } \operatorname{sig}(A) \text { is even } \\ \pm 1, & \text { if } \operatorname{sig}(A) \text { is odd }\end{cases}
$$

The following more general formula was given by Borcherds.

Proposition 2.2. (See [Bo2] Theorem 5.4.) Let $g=\left(\left(\begin{array}{ll}a & b \\ c & d\end{array}\right), \sqrt{c \tau+d}\right) \in \tilde{\Gamma}$, and suppose that $b$ and $c$ are divisible by $N$. Then

$$
\rho_{A}(g) \mathfrak{e}_{\lambda}=\chi_{A}(g) \mathfrak{e}_{d \lambda}
$$

Here $\chi_{A}$ denotes the character of $\tilde{\Gamma}_{0}(N)$ defined in Bo2] Theorem 5.4.

Lemma 2.3. Let $U=\left(\left(\begin{array}{ll}1 & 0 \\ 1 & 1\end{array}\right), \sqrt{\tau+1}\right) \in \tilde{\Gamma}$. The action of $U^{m}$ is given by

$$
\rho_{A}\left(U^{m}\right) \mathfrak{e}_{\lambda}=\frac{1}{|A|} \sum_{\mu, \nu \in A} e\left(-m \mu^{2} / 2+(\mu, \lambda-\nu)\right) \mathfrak{e}_{\nu}
$$

Proof. Since $U^{m}=S T^{-m} S^{-1}$, this follows immediately from (2.3) and (2.4).

In many recent works vector valued modular forms associated to the Weil representation are considered (see e.g. [Bo1], [Bo2], [Br], [McG], [Sch]). Let $k \in \frac{1}{2} \mathbb{Z}$, and let $\Gamma^{\prime} \subset \tilde{\Gamma}$ be a subgroup of finite index. A holomorphic function $f: \mathbb{H} \rightarrow \mathbb{C}[A]$ is called a modular form of weight $k$ and type $\rho_{A}$ for the group $\Gamma^{\prime}$, if

$$
f(M \tau)=\phi(\tau)^{2 k} \rho_{A}(M, \phi) f(\tau)
$$

for all $(M, \phi) \in \Gamma^{\prime}$, and $f$ is holomorphic at the cusps of $\Gamma^{\prime}$. We denote the $\mathbb{C}$-vector space of such holomorphic modular forms by $M_{k, A}\left(\Gamma^{\prime}\right)$. Moreover, for the full modular group we put $M_{k, A}=M_{k, A}(\tilde{\Gamma})$. Formula (2.5) implies that $M_{k, A}=\{0\}$ unless

$$
2 k \equiv \operatorname{sig}(A) \bmod 2 .
$$

Recall that for $f, g \in M_{k, A}\left(\Gamma^{\prime}\right)$ the Petersson scalar product is defined by

$$
(f, g)=\frac{1}{\left[\tilde{\Gamma}: \Gamma^{\prime}\right]} \int_{\Gamma^{\prime} \backslash \mathbb{H}}\langle f(\tau), g(\tau)\rangle y^{k} \frac{d x d y}{y^{2}} .
$$

Here $x$ denotes the real part and $y$ the imaginary part of $\tau \in \mathbb{H}$. The Petersson scalar product converges when $f \otimes g$ is a cusp form. 


\section{Extending the WeIL REPRESENTAtion}

In the classical theory of scalar valued modular forms Hecke operators play an important role (see e.g. Sh1]). It is natural to try to define Hecke operators on vector valued modular forms of type $\rho_{A}$ as well. This requires the extension of the representation $\rho_{A}$ to a representation (of a sufficiently large subgroup) of $\widetilde{\mathrm{GL}}_{2}^{+}(\mathbb{Q})$. However, it is not obvious how this can be done.

A natural starting point is to try to extend $\rho_{A}$, viewed as a representation of $S(N)$ (respectively $S_{1}(N)$ ), to a representation of (a double cover of) $\mathrm{GL}_{2}(\mathbb{Z} / N \mathbb{Z}$ ). However, it was observed by E. Freitag that such an extension does not exist in general. This follows from the following example.

Example 3.1. Let $d \equiv 1(\bmod 4)$ be an integer such that $p:=|d|$ is a prime. We consider the ring of integers $\mathcal{O}$ in the quadratic field $\mathbb{Q}(\sqrt{d})$ of discriminant $d$. Together with the norm form, it is an even lattice of type $(1,1)$ if $d>0$, and of type $(2,0)$ if $d<0$. The dual lattice is $\frac{1}{\sqrt{d}} \mathcal{O}$, the inverse of the different, and the corresponding discriminant form $A$ can be identified with the finite field $\mathbb{F}_{p}$ together with the quadratic form $x \mapsto-\frac{1}{d} x^{2}$. The associated Weil representation $\rho_{A}$ is a $p$-dimensional representation of $S(p)=\mathrm{SL}_{2}\left(\mathbb{F}_{p}\right)$ on $\mathbb{C}[A]$. The action of the orthogonal group $\mathrm{O}(A)=\{ \pm 1\}$ splits $\mathbb{C}[A]$ into two $S(p)$-invariant subspaces

$$
\begin{aligned}
& \mathbb{C}[A]^{+}=\operatorname{span}\left\{\mathfrak{e}_{\lambda}+\mathfrak{e}_{-\lambda} ; \lambda \in A\right\}, \\
& \mathbb{C}[A]^{-}=\operatorname{span}\left\{\mathfrak{e}_{\lambda}-\mathfrak{e}_{-\lambda} ; \lambda \in A\right\} .
\end{aligned}
$$

They have dimension $\frac{p+1}{2}$, and $\frac{p-1}{2}$, respectively. It follows from [NW], Theorem 4, that the corresponding representations of $S(p)$ are irreducible.

On the other hand, the character table of $\mathrm{GL}_{2}\left(\mathbb{F}_{p}\right)$ is well known, see e.g. [FH] $\S 5.2$. It has $p-1$ one-dimensional representations, $p-1$ irreducible $p$-dimensional representations, $(p-1)(p-2) / 2$ irreducible $(p+1)$-dimensional representations, and $\left(p^{2}-p\right) / 2$ irreducible $(p-1)$-dimsional representations.

Now assume that $p \geq 5$ and that $\rho_{A}$ has an extension $\tilde{\rho}_{A}$ to a representation of $\mathrm{GL}_{2}\left(\mathbb{F}_{p}\right)$. Because of the irreducibility of $\mathbb{C}[A]^{ \pm}$, such an extension would have to be a $p$-dimensional irreducible representation of $\mathrm{GL}_{2}\left(\mathbb{F}_{p}\right)$. But these representations remain irreducible under restriction to $\mathrm{SL}_{2}\left(\mathbb{F}_{p}\right)$, see $[\mathrm{FH}]$, p.72 (2). We obtain a contradiction.

Remark 3.2. In [McG], McGraw continues $\rho_{A}$ to an action of $\mathrm{GL}_{2}(\mathbb{Z} / N \mathbb{Z})$. However, this action is not $\mathbb{C}$-linear, causing serious difficulties when one tries to define Hecke operators.

Here we consider a different group extension $Q(N)$ of $S(N)$ and show that $\rho_{A}$ can be continued to a representation of $Q(N)$. Together with the considerations of Section 5 this will suffice for many applications of Hecke operators; for instance, to define the standard $L$-function of a modular form of type $\rho_{A}$.

Let $A$ be a discriminant form as in the previous section, and let $N$ be the level of $A$. We denote by $U(N)$ the unit group of $\mathbb{Z} / N \mathbb{Z}$. We briefly write $G(N)=\mathrm{GL}_{2}(\mathbb{Z} / N \mathbb{Z})$ for the general linear group modulo $N$. The determinant homomorphism $G(N) \rightarrow U(N)$ gives 
rise to the exact sequence

$$
1 \longrightarrow S(N) \longrightarrow G(N) \longrightarrow U(N) \longrightarrow 1 \text {. }
$$

This sequence splits and $G(N)$ can be viewed as a semidirect product of $S(N)$ and $U(N)$.

3.1. The case of even signature. Throughout this subsection we assume that $\operatorname{sig}(A)$ is even. Let $Q(N)$ be the group

$$
Q(N)=\left\{(M, r) \in G(N) \times U(N) ; \quad \operatorname{det}(M) \equiv r^{2} \quad(\bmod N)\right\}
$$

with the product defined component-wise. We have an exact sequence

$$
1 \longrightarrow S(N) \longrightarrow Q(N) \longrightarrow U(N) \longrightarrow 1,
$$

where $S(N) \rightarrow Q(N)$ is given by $M \mapsto(M, 1)$, and $Q(N) \rightarrow U(N)$ is given by $(M, r) \mapsto r$. The latter homomorphism has the section

$$
U(N) \longrightarrow Q(N), \quad r \mapsto\left(\left(\begin{array}{ll}
r & 0 \\
0 & r
\end{array}\right), r\right)
$$

For $(M, r) \in Q(N)$ the assignment $(M, r) \mapsto\left(M\left(\begin{array}{cc}r & 0 \\ 0 & r\end{array}\right)^{-1}, r\right)$ defines an isomorphism $Q(N) \cong S(N) \times U(N)$.

We consider the action of $S(N)$ on $\mathbb{C}[A]$ by the Weil representation $\rho_{A}$. In view of (2.12), the assignment $r \mapsto \frac{g(A)}{g_{r}(A)}$ defines a character of $U(N)$. We define a unitary representation of $U(N)$ on $\mathbb{C}[A]$ by putting

$$
\rho_{A}(r) \mathfrak{e}_{\lambda}=\rho_{A}\left(\left(\begin{array}{ll}
r & 0 \\
0 & r
\end{array}\right), r\right) \mathfrak{e}_{\lambda}=\frac{g(A)}{g_{r}(A)} \mathfrak{e}_{\lambda}
$$

for $r \in U(N)$.

Proposition 3.3. The Weil representation $\rho_{A}$ of the group $S(N)$ extends to a unitary representation of $Q(N)$ by (3.5).

Proof. Since $Q(N)=S(N) \times U(N)$ we only have to check that the actions of $S(N)$ and $U(N)$ commute. This is obvious.

Remark 3.4. Clearly we could take any character of $U(N)$ to define the action of the Weil representation on $U(N)$. The above choice is compatible with the definition of the Weil representation on double cosets in Section 5. Moreover, it is compatible with the usual Hecke operators on scalar valued modular forms, see Remark 4.4 and Remark 4.11.

The following proposition shows that the first entry of an element $(M, r) \in Q(N)$ gives the "essential contribution" to the Weil representation. If we fix $M$ then for the different choices of $r \in U(N)$ the Weil representation $\rho_{A}(M, r)$ differs by the action of an element of the orthogonal group $\mathrm{O}(A)$.

Proposition 3.5. Let $\left(M, r_{1}\right),\left(M, r_{2}\right) \in Q(N)$. Then $h: A \rightarrow A, \lambda \mapsto r_{1} r_{2}^{-1} \lambda$ is an orthogonal transformation in $\mathrm{O}(A)$, and

$$
\rho_{A}\left(M, r_{1}\right) \mathfrak{e}_{\lambda}=\rho_{A}\left(M, r_{2}\right) \rho_{A}(h) \mathfrak{e}_{\lambda}=\rho_{A}\left(M, r_{2}\right) \mathfrak{e}_{h^{-1} \lambda}
$$


Proof. We have $\left(M, r_{2}\right)^{-1}\left(M, r_{1}\right)=\left(1, r_{1} r_{2}^{-1}\right)$, and $t=r_{1} r_{2}^{-1} \in U(N)$ has the property that $t^{2} \equiv 1(\bmod N)$. According to (2.10) and (3.5), the action of $(1, t)$ is given by

$$
\rho_{A}(1, t) \mathfrak{e}_{\lambda}=\mathfrak{e}_{t^{-1} \lambda}
$$

Since $(t \lambda, t \mu)=\left(t^{2} \lambda, \mu\right)=(\lambda, \mu)$ for all $\lambda, \mu \in A$, multiplication by $t^{-1}$ is an orthogonal transformation.

Lemma 3.6. We have

$$
\begin{gathered}
\rho_{A}\left(\left(\begin{array}{cc}
r^{2} & 0 \\
0 & 1
\end{array}\right), r\right) \mathfrak{e}_{\lambda}=\mathfrak{e}_{r^{-1} \lambda} \\
\rho_{A}\left(\left(\begin{array}{cc}
1 & 0 \\
0 & r^{2}
\end{array}\right), r\right) \mathfrak{e}_{\lambda}=\mathfrak{e}_{r \lambda} .
\end{gathered}
$$

Proof. In $Q(N)$ we have

$$
\left(\left(\begin{array}{cc}
r^{2} & 0 \\
0 & 1
\end{array}\right), r\right)=\left(R_{r^{-1}}, 1\right)\left(\left(\begin{array}{ll}
r & 0 \\
0 & r
\end{array}\right), r\right)
$$

Therefore the fist formula follows from Lemma 2.1 and (3.5). The second formula follows similarly.

3.2. The case of odd signature. Throughout this subsection we assume that $\operatorname{sig}(A)$ is odd. In this case the argument of the previous section only shows that the Weil representation extends to a projective representation of $Q(N)$. More precisely, it extends to a group homomorphism

$$
Q(N) \longrightarrow \mathrm{U}(\mathbb{C}[A]) /\{ \pm 1\}
$$

This projective representation gives rise to a 2-cocycle of $Q(N)$ with values in $\{ \pm 1\}$. The cocycle defines a central extension $Q_{1}(N)$ of $Q(N)$ by $\{ \pm 1\}$. The group $S_{1}(N)$, see (2.8), can be identified with a subgroup of $Q_{1}(N)$ (see Section 4.2 for more details).

Proposition 3.7. The assignment (3.5) defines an extension of the Weil representation to a projective representation of $Q(N)$. It lifts to a unitary representation of $Q_{1}(N)$.

\section{HeCKe OPERATORS ON VECTOR VALUED MODUlaR FORMS}

We now use the results of Section 3 to define Hecke operators on vector valued modular forms of type $\rho_{A}$. We consider the groups

$$
\begin{gathered}
\mathcal{G}(N)=\left\{M \in \mathrm{GL}_{2}^{+}(\mathbb{Q}) ; \quad \exists n \in \mathbb{Z} \text { with }(n, N)=1 \text { such that } n M \in \mathrm{M}_{2}(\mathbb{Z})\right. \\
\text { and }(\operatorname{det}(n M), N)=1\}, \\
\mathcal{Q}(N)=\left\{(M, r) \in \mathcal{G}(N) \times U(N) ; \quad \operatorname{det}(M) \equiv r^{2} \quad(\bmod N)\right\} .
\end{gathered}
$$

We view $\Gamma$ as a subgroup of $\mathcal{Q}(N)$ by the embedding $M \mapsto(M, 1)$. We are interested in the action of the Hecke algebra of the pair $(\mathcal{Q}(N), \Gamma)$ on modular forms of type $\rho_{A}$ and weight $k$. We have to distinguish the cases whether $\operatorname{sig}(A)$ is even or odd. 
4.1. The case of even signature. The composition of the reduction map $\mathcal{Q}(N) \rightarrow Q(N)$ with the Weil representation $\rho_{A}: Q(N) \rightarrow \mathrm{U}(\mathbb{C}[A])$ induces a unitary representation of $\mathcal{Q}(N)$ on $\mathbb{C}[A]$, which we will denote by $\rho_{A}$ as well. This left action induces a corresponding right action by

$$
\left.\mathfrak{a}\right|_{A}(M, r)=\rho_{A}(M, r)^{-1} \mathfrak{a} .
$$

In view of (2.14) we only have to consider modular forms of integral weight $k \in \mathbb{Z}$. Let $f$ be a complex valued function on $\mathbb{H}$. For $M \in \mathrm{GL}_{2}^{+}(\mathbb{R})$ the Petersson slash operator is defined by

$$
\left(\left.f\right|_{k} M\right)(\tau)=\operatorname{det}(M)^{k / 2} j(M, \tau)^{-k} f(M \tau) .
$$

This defines a right action of $\mathcal{G}(N)$ on functions $\mathbb{H} \rightarrow \mathbb{C}$. The center acts by multiplication with \pm 1 .

If $f: \mathbb{H} \rightarrow \mathbb{C}[A]$ is a function we write $f=\sum_{\lambda \in A} f_{\lambda} \otimes \mathfrak{e}_{\lambda}$ for its decomposition in components with respect to the standard basis of $\mathbb{C}[A]$. The tensor product of the above two actions yields a right action of $\mathcal{Q}(N)$ on such functions, denoted by

$$
\left.f\right|_{k, A}(M, r)=\sum_{\lambda \in A}\left(\left.f_{\lambda}\right|_{k} M\right) \otimes\left(\left.\mathfrak{e}_{\lambda}\right|_{A}(M, r)\right) .
$$

Notice that a holomorphic function $f: \mathbb{H} \rightarrow \mathbb{C}[A]$ belongs to $M_{k, A}$, if and only if

$$
\left.f\right|_{k, A} M=f
$$

for all $M \in \Gamma$, and $f$ is holomorphic at the cusp $\infty$.

We consider the Hecke algebra of the pair $(\mathcal{Q}(N), \Gamma)$ in the sense of Shimura [Sh1]. If $(M, r) \in \mathcal{Q}(N)$, the corresponding double coset decomposes into a finite union of left cosets

$$
\Gamma \cdot(M, r) \cdot \Gamma=\bigcup_{\gamma \in \Gamma \backslash \Gamma M \Gamma} \Gamma \cdot(\gamma, r) .
$$

Definition 4.1. For $(M, r) \in \mathcal{Q}(N)$ we define the corresponding Hecke operator $T(M, r)$ on $M_{k, A}$ by

$$
\left.f\right|_{k, A} T(M, r)=\left.\operatorname{det}(M)^{k / 2-1} \sum_{\gamma \in \Gamma \backslash \Gamma M \Gamma} f\right|_{k, A}(\gamma, r), \quad f \in M_{k, A} .
$$

The usual argument now shows that $\left.f\right|_{k, A} T(M, r) \in M_{k, A}$. Hence $T(M, r)$ defines an endomorphism of $M_{k, A}$. A modular form $f \in M_{k, A}$ has a Fourier expansion of the form

$$
f(\tau)=\sum_{\lambda \in A} \sum_{n \in \mathbb{Z}+\lambda^{2} / 2} c(\lambda, n) e(n \tau) \otimes \mathfrak{e}_{\lambda}
$$

Theorem 4.2. Let $p$ be a prime which is a square modulo $N$, and assume that $r \in U(N)$ with $p \equiv r^{2}(\bmod N)$. Let $f \in M_{k, A}$ and denote the Fourier expansion as in (4.7). Then

$$
\left.f\right|_{k, A} T\left(\left(\begin{array}{ll}
p & 0 \\
0 & 1
\end{array}\right), r\right)=\sum_{\lambda \in A} \sum_{n \in \mathbb{Z}+\lambda^{2} / 2} b(\lambda, n) e(n \tau) \otimes \mathfrak{e}_{\lambda},
$$


where

$$
b(\lambda, n)=c(r \lambda, p n)+p^{k-1} c(\lambda / r, n / p) .
$$

Here we understand that $c(\lambda / r, n / p)=0$ if $p \nmid n$, i.e., if $\operatorname{ord}_{p}(n)=0$.

Proof. Using Lemma 3.6, the formula follows in the same way as in the scalar valued case.

Proposition 4.3. Let $p$ be a prime coprime to $N$. Let $f \in M_{k, A}$ and denote the Fourier expansion as in (4.7). Then

$$
\left.f\right|_{k, A} T\left(\left(\begin{array}{cc}
p^{2} & 0 \\
0 & 1
\end{array}\right), p\right)=\sum_{\lambda \in A} \sum_{n \in \mathbb{Z}+\lambda^{2} / 2} b(\lambda, n) e(n \tau) \otimes \mathfrak{e}_{\lambda},
$$

where

$$
b(\lambda, n)=c\left(p \lambda, p^{2} n\right)+\frac{g(A)}{g_{p}(A)} p^{k-2}\left(\delta_{p}(n)-1\right) c(\lambda, n)+p^{2 k-2} c\left(\lambda / p, n / p^{2}\right),
$$

and

$$
\delta_{p}(n)= \begin{cases}p, & \text { if } p \mid n, \\ 0, & \text { if } p \nmid n .\end{cases}
$$

Moreover, we understand that $c\left(\lambda / p, n / p^{2}\right)=0$ if $p^{2} \nmid n$.

Proof. We omit the proof, which is similar (but easier) to the proof of Theorem 4.10 ,

Remark 4.4. If $|A|=\ell$ is a prime, then $M_{k, A}$ can be identified with the plus or minus subspace of the space $M_{k}\left(\ell, \chi_{\ell}\right)$ of scalar valued modular forms for $\Gamma_{0}(\ell)$ with nebentypus, see $[\mathrm{BB}]$. Under this identification, the above Hecke operators correspond to the usual Hecke operators on $M_{k}\left(\ell, \chi_{\ell}\right)$. This follows by comparing the actions on Fourier expansions. For Hecke operators on $M_{k}\left(\ell, \chi_{\ell}\right)$ see e.g. [Mi], Lemma 4.5.14.

4.2. The case of odd signature. We now assume that $\operatorname{sig}(A)$ is odd. In view of (2.14) we only have to consider modular forms of half integral weight $k \in \mathbb{Z}+1 / 2$. In this case, the problem arises that both, (4.3) and (4.4), only define projective actions of $\mathcal{Q}(N)$. To obtain honest actions, one has to consider appropriate central extensions.

We begin by considering the action on $\mathbb{C}[A]$. The composition of the natural reduction $\mathcal{Q}(N) \rightarrow Q(N)$ with the projective Weil representation $\rho_{A}: Q(N) \rightarrow \mathrm{U}(\mathbb{C}[A]) /\{ \pm 1\}$ induces a projective representation

$$
\rho_{A}: \mathcal{Q}(N) \longrightarrow \mathrm{U}(\mathbb{C}[A]) /\{ \pm 1\}, \quad g \mapsto \rho_{A}(g)
$$

If we choose for every $g \in \mathcal{Q}(N)$ a $\tilde{\rho}_{A}(g) \in \mathrm{U}(\mathbb{C}[A])$ such that $\tilde{\rho}_{A}(g) \mapsto \rho_{A}(g)$ under the projection to $\mathrm{U}(\mathbb{C}[A]) /\{ \pm 1\}$, we obtain a 2-cocycle $c$ with values in $\{ \pm 1\}$ defined by

$$
\tilde{\rho}_{A}\left(g_{1} g_{2}\right)=c\left(g_{1}, g_{2}\right) \tilde{\rho}_{A}\left(g_{1}\right) \tilde{\rho}_{A}\left(g_{2}\right)
$$

for $g_{1}, g_{2} \in \mathcal{Q}(N)$. This cocycle gives rise to a central extension

$$
\mathcal{Q}_{1}(N)=\mathcal{Q}(N) \times\{ \pm 1\}
$$


where the multiplication is defined by

$$
\left(g_{1}, t_{1}\right)\left(g_{2}, t_{2}\right)=\left(g_{1} g_{2}, t_{1} t_{2} c\left(g_{1}, g_{2}\right)^{-1}\right) \text {. }
$$

Here the cocycle condition

$$
c\left(g_{1} g_{2}, g_{3}\right) c\left(g_{1}, g_{2}\right)=c\left(g_{1}, g_{2} g_{3}\right) c\left(g_{2}, g_{3}\right)
$$

is equivalent to the associativity law for the above multiplication. We obtain a unitary representation

$$
\mathcal{Q}_{1}(N) \longrightarrow \mathrm{U}(\mathbb{C}[A])
$$

by putting $\rho_{A}(g, t)=t \tilde{\rho}_{A}(g)$. This left action induces a corresponding right action

$$
\left.\mathfrak{a}\right|_{A}(g, t)=\rho_{A}(g, t)^{-1} \mathfrak{a},
$$

for $(g, t) \in \mathcal{Q}_{1}(N)$ and $\mathfrak{a} \in \mathbb{C}[A]$.

Without loss of generality, for $(M, 1) \in \Gamma \times\{1\} \subset \mathcal{Q}(N)$ we choose

$$
\tilde{\rho}_{A}(M, 1)=\rho_{A}(M, \sqrt{j(M, \tau)}) .
$$

Then we have an injective homomorphism

$$
\tilde{\Gamma} \longrightarrow \mathcal{Q}_{1}(N), \quad(M, \pm \sqrt{j(M, \tau)}) \mapsto(M, 1, \pm 1)
$$

Moreover, for a positive integer $m$ coprime to $N$, we put

$$
\tilde{\rho}_{A}\left(\left(\begin{array}{cc}
m^{2} & 0 \\
0 & 1
\end{array}\right), m\right) \mathfrak{e}_{\lambda}=\mathfrak{e}_{m^{-1} \lambda}
$$

To define an action on functions we consider the metaplectic group $\widetilde{G L}_{2}^{+}(\mathbb{R})$. It acts on functions $f: \mathbb{H} \rightarrow \mathbb{C}$ by

$$
\left(\left.f\right|_{k}(M, \phi)\right)(\tau)=\operatorname{det}(M)^{k / 2} \phi(\tau)^{-2 k} f(M \tau)
$$

for $(M, \phi) \in{\widetilde{\mathrm{GL}_{2}}}_{2}^{+}(\mathbb{R})$. In particular, we have an action of $\widetilde{\mathcal{H}}(N) \subset \widetilde{\mathrm{GL}}_{2}^{+}(\mathbb{R})$, where

$$
\mathcal{H}(N)=\{M \in \mathcal{G}(N) ; \quad \operatorname{det}(M) \text { is a square } \bmod N\} \subset \mathrm{GL}_{2}^{+}(\mathbb{R})
$$

is the image of the projection of $\mathcal{Q}(N)$ to the first component. Notice that the cocycle of $\mathcal{Q}(N)$ given by the choice of $\pm \sqrt{j(M, \tau)}$ for $(M, r) \in \mathcal{Q}(N)$ and the cocycle $c$ are not isomorphic (this follows for instance from Lemma 4.8 below). However, their restrictions to $\tilde{\Gamma}$ are isomorphic.

To define an action on $\mathbb{C}[A]$-valued functions in weight $k$ we have to consider a combination of the above extensions. We let $\mathcal{Q}_{2}(N)$ be the group of tuples $(M, \phi, r, t)$, where $g=(M, \phi) \in \widetilde{\mathcal{H}}(N)$, and $r \in U(N)$ with $\operatorname{det}(M) \equiv r^{2}(\bmod N)$, and $t \in\{ \pm 1\}$. The composition law is defined by

$$
\left(g_{1}, r_{1}, t_{1}\right)\left(g_{2}, r_{2}, t_{2}\right)=\left(g_{1} g_{2}, r_{1} r_{2}, t_{1} t_{2} c\left(\left(M_{1}, r_{1}\right),\left(M_{2}, r_{2}\right)\right)^{-1}\right)
$$

for $\left(g_{i}, r_{i}, t_{i}\right) \in \mathcal{Q}_{2}(N)$ and $g_{i}=\left(M_{i}, \phi_{i}\right)$. We denote by $P: \mathcal{Q}_{2} \rightarrow \widetilde{\mathcal{H}}(N)$ the natural projection. It has the kernel

$$
\left\{(1,1, r, t) \in \mathcal{Q}_{2} ; r^{2} \equiv 1(N)\right\} .
$$


Over $\tilde{\Gamma}$ the projection $P$ has the section

$$
L: \tilde{\Gamma} \longrightarrow \mathcal{Q}_{2}, \quad(M, \pm \sqrt{j(M, \tau)}) \mapsto(M, \pm \sqrt{j(M, \tau)}, 1, \pm 1) .
$$

We write

$$
\Delta=L(\tilde{\Gamma})
$$

We define the Weil representation $\rho_{A}$ on $\mathcal{Q}_{2}(N)$ by composing the natural map to $\mathcal{Q}_{1}(N)$ with the Weil representation on that group. For $\gamma \in \tilde{\Gamma}$ we have

$$
\rho_{A}(\gamma)=\rho_{A}(L(\gamma))
$$

The tensor product of the above two actions yields a right action of $\mathcal{Q}_{2}(N)$ on functions $f: \mathbb{H} \rightarrow \mathbb{C}[A]$. If we write $f=\sum_{\lambda \in A} f_{\lambda} \otimes \mathfrak{e}_{\lambda}$ for the decomposition in components with respect to the standard basis of $\mathbb{C}[A]$, the action is given by

$$
\left.f\right|_{k, A}(M, \phi, r, t)=\sum_{\lambda \in A}\left(\left.f_{\lambda}\right|_{k}(M, \phi)\right) \otimes\left(\left.\mathfrak{e}_{\lambda}\right|_{A}(M, r, t)\right)
$$

for $(M, \phi, r, t) \in \mathcal{Q}_{2}(N)$. A holomorphic function $f: \mathbb{H} \rightarrow \mathbb{C}[A]$ belongs to $M_{k, A}$, if and only if $\left.f\right|_{k, A}(M, \phi)=f$ for all $(M, \phi) \in \tilde{\Gamma}$, and $f$ is holomorphic at the cusp $\infty$.

We may now define Hecke operators on modular forms of type $\rho_{A}$ following Shimura [Sh2]. For $\alpha=(M, \phi) \in \widetilde{\mathcal{H}}(N)$ and $\xi=(\alpha, r, t) \in \mathcal{Q}_{2}(N)$ we consider the double coset $\Delta \xi \Delta$. If

$$
\Delta \xi \Delta=\bigcup_{i} \Delta \xi_{i}
$$

is a left coset decomposition, we define the Hecke operator $T(\xi)$ by

$$
\left.f\right|_{k, A} T(\xi)=\left.\operatorname{det}(\alpha)^{k / 2-1} \sum_{i} f\right|_{k, A} \xi_{i}
$$

for $f \in M_{k, A}$. It is easily seen that $T(\xi)$ is independent of the choice of the coset representatives and defines an endomorphism of $M_{k, A}$. We recall the following standard lemma.

Lemma 4.5. Let the notation be as above. Then

$$
\Delta=\bigcup_{i \in I}\left(\Delta \cap \xi^{-1} \Delta \xi\right) \cdot \gamma_{i}
$$

$\left(\gamma_{i} \in \Delta\right)$ is a disjoint left coset decomposition if and only if

$$
\Delta \xi \Delta=\bigcup_{i \in I} \Delta \cdot \xi \gamma_{i}
$$

is a disjoint left coset decomposition.

To compute the action of Hecke operators we have to compare the groups $\Delta \cap \xi^{-1} \Delta \xi$ and $L\left(\tilde{\Gamma} \cap \alpha^{-1} \tilde{\Gamma} \alpha\right)$. Let $\alpha \in \tilde{\mathcal{H}}(N)$ and $\xi \in \mathcal{Q}_{2}(N)$ with $P(\xi)=\alpha$. For $\gamma \in \tilde{\Gamma} \cap \alpha^{-1} \tilde{\Gamma} \alpha$ we have

$$
L\left(\alpha \gamma \alpha^{-1}\right)=\xi L(\gamma) \xi^{-1} \cdot(1,1, t(\gamma))
$$


with $t(\gamma) \in\{ \pm 1\}$. Here $t(\gamma)$ is independent of the choice of $\xi$ and is determined by the condition

$$
\rho_{A}\left(\alpha \gamma \alpha^{-1}\right)=t(\gamma) \rho_{A}(\xi) \rho_{A}(\gamma) \rho_{A}(\xi)^{-1}
$$

Hence $t$ defines a group homomorphism

$$
t: \tilde{\Gamma} \cap \alpha^{-1} \tilde{\Gamma} \alpha \longrightarrow\{ \pm 1\} .
$$

Lemma 4.6. Let the notation be as above. We have $L(\operatorname{ker}(t))=\Delta \cap \xi^{-1} \Delta \xi$. Moreover, if $t$ is non-trivial, then $\left.f\right|_{k, A} T(\xi)=0$ for all $f \in M_{k, A}$.

Proof. The proof is analogous to Proposition 1.0 in [Sh2]

Lemma 4.7. Let the notation be as above. The homomorphism $t$ is trivial if and only if $P$ gives a bijective map of $\Delta \xi \Delta$ onto $\tilde{\Gamma} \alpha \tilde{\Gamma}$. Moreover, when this is the case then $\Delta \xi \Delta=\bigcup_{i} \Delta \xi_{i}$ (where $\xi_{i} \in \Delta \xi \Delta$ ) is a disjoint union if and only if $\tilde{\Gamma} \alpha \tilde{\Gamma}=\bigcup_{i} \tilde{\Gamma} P\left(\xi_{i}\right)$ is a disjoint union.

Proof. The proof is analogous to Proposition 1.1 in [Sh2]

Lemma 4.8. Let $m, n$ be positive integers coprime to $N, \alpha=\left(\left(\begin{array}{cc}m & 0 \\ 0 & n\end{array}\right), \sqrt{n}\right) \in \widetilde{\mathcal{H}}(N)$, and $\xi=(\alpha, r, t) \in \mathcal{Q}_{2}(N)$. Define $t: \tilde{\Gamma} \cap \alpha^{-1} \tilde{\Gamma} \alpha \longrightarrow\{ \pm 1\}$ as in (4.24). Then

$$
t\left(\left(\begin{array}{ll}
a & b \\
c & d
\end{array}\right), \sqrt{c \tau+d}\right)=\left(\frac{m n}{d}\right)
$$

Here the quadratic residue symbol is defined as in [Sh2].

Proof. Define $\Gamma^{\prime}=\Gamma_{0}(m) \cap \Gamma^{0}(n) \subset \Gamma$. We first notice that

$$
\tilde{\Gamma}^{\prime}=\tilde{\Gamma} \cap \alpha^{-1} \tilde{\Gamma} \alpha
$$

Since $t$ is a homomorphism it suffices to prove the assertion for a set of generators of $\tilde{\Gamma}^{\prime}$. It is easily verified that $\tilde{\Gamma}^{\prime}$ is generated by $T^{n}, U^{m}$, and $\tilde{\Gamma}^{\prime} \cap \tilde{\Gamma}_{0}^{0}(N)$.

For $\gamma=T^{n} \in \tilde{\Gamma}^{\prime}$ we have $\alpha \gamma \alpha^{-1}=T^{m}$. We compute $t(\gamma)$ using (4.23). There is a constant $C_{\xi}$ of modulus 1 such that $\rho_{A}(\xi) \mathfrak{e}_{\lambda}=C_{\xi} \mathfrak{e}_{n r^{-1} \lambda}$. Hence

$$
\begin{aligned}
\rho_{A}(\xi) \rho_{A}\left(T^{n}\right) \rho_{A}(\xi)^{-1} \mathfrak{e}_{\lambda} & =C_{\xi}^{-1} \rho_{A}(\xi) \rho_{A}\left(T^{n}\right) \mathfrak{e}_{n^{-1} r \lambda} \\
& =C_{\xi}^{-1} e\left(n^{-1} r^{2} \lambda^{2} / 2\right) \rho_{A}(\xi) \mathfrak{e}_{n^{-1} r \lambda} \\
& =e\left(n^{-1} r^{2} \lambda^{2} / 2\right) \mathfrak{e}_{\lambda} \\
& =e\left(m \lambda^{2} / 2\right) \mathfrak{e}_{\lambda} .
\end{aligned}
$$

Here $n^{-1}$ in the exponentials means the inverse of $n$ in $U(N)$. On the other hand, we have $\rho_{A}\left(T^{m}\right) \mathfrak{e}_{\lambda}=e\left(m \lambda^{2} / 2\right) \mathfrak{e}_{\lambda}$. Hence $t\left(T^{n}\right)=1$ in accordance with the formula. 
For $\gamma=U^{m} \in \tilde{\Gamma}^{\prime}$ we have $\alpha \gamma \alpha^{-1}=U^{n}$. We find

$$
\begin{aligned}
\rho_{A}(\xi) \rho_{A}\left(U^{m}\right) \rho_{A}(\xi)^{-1} \mathfrak{e}_{\lambda} & =C_{\xi}^{-1} \rho_{A}(\xi) \rho_{A}\left(U^{m}\right) \mathfrak{e}_{n^{-1} r \lambda} \\
& =\frac{C_{\xi}^{-1}}{|A|} \rho_{A}(\xi) \sum_{\mu, \nu \in A} e\left(-m \mu^{2} / 2+\left(\mu, n^{-1} r \lambda-\nu\right)\right) \mathfrak{e}_{\nu} \\
& =\frac{1}{|A|} \sum_{\mu, \nu \in A} e\left(-m \mu^{2} / 2+\left(n^{-1} r \mu, \lambda-\nu\right)\right) \mathfrak{e}_{\nu} \\
& =\frac{1}{|A|} \sum_{\mu, \nu \in A} e\left(-n \mu^{2} / 2+(\mu, \lambda-\nu)\right) \mathfrak{e}_{\nu} .
\end{aligned}
$$

This is equal to $\rho_{A}\left(U^{n}\right)$. Hence $t\left(U^{m}\right)=1$ in accordance with the formula.

To compute $t(\gamma)$ for $\gamma \in \tilde{\Gamma}^{\prime} \cap \tilde{\Gamma}_{0}^{0}(N)$, we use the formula for the Weil representation of Proposition 2.2. Using the definition one easily checks that if $\gamma \in \tilde{\Gamma}^{\prime} \cap \tilde{\Gamma}_{0}^{0}(N)$, we have

$$
\chi_{A}(\gamma)=\chi_{A}\left(\alpha \gamma \alpha^{-1}\right)\left(\frac{m n}{d}\right)
$$

Therefore

$$
\begin{aligned}
\rho_{A}(\xi) \rho_{A}(\gamma) \rho_{A}(\xi)^{-1} \mathfrak{e}_{\lambda} & =C_{\xi}^{-1} \rho_{A}(\xi) \rho_{A}(\gamma) \mathfrak{e}_{n^{-1} r \lambda} \\
& =\chi_{A}(\gamma) \mathfrak{e}_{d \lambda} \\
& =\left(\frac{m n}{d}\right) \rho_{A}\left(\alpha \gamma \alpha^{-1}\right) \mathfrak{e}_{\lambda}
\end{aligned}
$$

So $t(\gamma)=\left(\frac{m n}{d}\right)$ as claimed.

Proposition 4.9. Let $\alpha=(M, \phi) \in \widetilde{\mathcal{H}}(N)$ and $\xi=(\alpha, r, t) \in \mathcal{Q}_{2}(N)$. Then the Hecke operator $T(\xi)$ on $M_{k, A}$ vanishes identically unless $\operatorname{det}(M)$ is a square in $\mathbb{Q}$.

Proof. By multiplying with a positive integer we may assume without loss of generality that $M$ has entries in $\mathbb{Z}$. According to the elementary divisor theorem for $\Gamma$ we may further assume that $M=\left(\begin{array}{cc}m & 0 \\ 0 & n\end{array}\right)$ with positive integers $m, n$. So we may assume that

$$
\alpha=\left(\left(\begin{array}{cc}
m & 0 \\
0 & n
\end{array}\right), \sqrt{n}\right) .
$$

Hence the assertion follows from Lemma 4.6 and Lemma 4.8.

We now study the relation between Hecke operators and Fourier coefficients. The following theorem is the analogue of Proposition 4.3 in the odd signature case. It can be viewed as a generalization of Theorem 1.7 in [Sh2].

Theorem 4.10. Let $p$ be a prime coprime to $N$, and put

$$
\begin{aligned}
& \alpha=\left(\left(\begin{array}{ll}
p^{2} & 0 \\
0 & 1
\end{array}\right), 1\right) \in \widetilde{\mathcal{H}}(N), \\
& \xi=(\alpha, p, 1) \in \mathcal{Q}_{2}(N) .
\end{aligned}
$$


Let $f \in M_{k, A}$ and write

$$
\begin{aligned}
f(\tau) & =\sum_{\lambda \in A} \sum_{n \in \mathbb{Z}+\lambda^{2} / 2} c(\lambda, n) e(n \tau) \otimes \mathfrak{e}_{\lambda}, \\
\left.f\right|_{k, A} T(\xi) & =\sum_{\lambda \in A} \sum_{n \in \mathbb{Z}+\lambda^{2} / 2} b(\lambda, n) e(n \tau) \otimes \mathfrak{e}_{\lambda} .
\end{aligned}
$$

Then

$$
b(\lambda, n)=c\left(p \lambda, p^{2} n\right)+\epsilon_{p}^{\operatorname{sig}(A)+\left(\frac{-1}{|A|}\right)}\left(\frac{p}{|A| 2^{\operatorname{sig}(A)}}\right) p^{k-3 / 2}\left(\frac{-n}{p}\right) c(\lambda, n)+p^{2 k-2} c\left(\lambda / p, n / p^{2}\right) .
$$

Here, for an odd integer $d$ we put

$$
\epsilon_{d}= \begin{cases}1, & \text { if } d \equiv 1 \quad(\bmod 4), \\ i, & \text { if } d \equiv-1 \quad(\bmod 4) .\end{cases}
$$

Moreover, we understand that $c\left(\lambda / p, n / p^{2}\right)=0$ if $p^{2} \nmid n$.

Proof. To compute $\left.f\right|_{k, A} T(\xi)$, we need a set of representatives for $\Delta \backslash \Delta \xi \Delta$. In view of Lemma 4.7 and Lemma 4.8, the map

$$
L_{\xi}: \tilde{\Gamma} \alpha \tilde{\Gamma} \longrightarrow \Delta \xi \Delta, \quad \delta=\gamma \alpha \gamma^{\prime} \mapsto L_{\xi}(\delta):=L(\gamma) \xi L\left(\gamma^{\prime}\right)
$$

is a bijection (where $\gamma, \gamma^{\prime} \in \tilde{\Gamma}$ ). Here we have $L_{\xi}(\delta)=(\delta, p, t)$, and $t=t(\delta)$ is uniquely determined by the condition

$$
\rho_{A}(\delta, p, t)=\rho_{A}(\gamma) \rho_{A}(\xi) \rho_{A}\left(\gamma^{\prime}\right) .
$$

We have the disjoint left coset decomposition

$$
\tilde{\Gamma} \alpha \tilde{\Gamma}=\tilde{\Gamma} \alpha \cup \bigcup_{h(p)^{*}} \tilde{\Gamma} \beta_{h} \cup \bigcup_{b\left(p^{2}\right)} \tilde{\Gamma} \gamma_{b}
$$

where

$$
\begin{aligned}
& \beta_{h}=\left(\left(\begin{array}{cc}
p & h N \\
0 & p
\end{array}\right), \sqrt{p}\right)=\left(\left(\begin{array}{cc}
r & N h \\
N s & p
\end{array}\right), \sqrt{N s \tau+p}\right) \alpha\left(\left(\begin{array}{cc}
1 & 0 \\
-N p s & 1
\end{array}\right), \sqrt{-N p s \tau+1}\right), \\
& \gamma_{b}=\left(\left(\begin{array}{cc}
1 & b \\
0 & p^{2}
\end{array}\right), p\right)=\left(\left(\begin{array}{cc}
d & N t \\
-N & p^{2}
\end{array}\right), \sqrt{-N \tau+p^{2}}\right) \alpha\left(\left(\begin{array}{cc}
1 & -N t \\
N & p^{2} d
\end{array}\right), \sqrt{N \tau+p^{2} d}\right) T^{b}
\end{aligned}
$$

and $r, s \in \mathbb{Z}$ are chosen such that $p r-N^{2} h s=1$, and $d, t \in \mathbb{Z}$ are chosen such that $p^{2} d+N^{2} t=1$. Consequently, we obtain the disjoint left coset decomposition

$$
\Delta \xi \Delta=\Delta \xi \cup \bigcup_{h(p)^{*}} \Delta L_{\xi}\left(\beta_{h}\right) \cup \bigcup_{b\left(p^{2}\right)} \Delta L_{\xi}\left(\gamma_{b}\right) .
$$


The action of $L_{\xi}\left(\beta_{h}\right)$ and $L_{\xi}\left(\gamma_{b}\right)$ in the Weil representation can be computed by means of (4.25) and the above decompositions. Using Proposition 2.2 and the fact that $\rho_{A}(\xi) \mathfrak{e}_{\lambda}=$ $\mathfrak{e}_{p^{-1} \lambda}$ by (4.13), we find that

$$
\begin{aligned}
\rho_{A}\left(L_{\xi}\left(\gamma_{b}\right)\right) \mathfrak{e}_{\lambda}= & \chi_{A}\left(\left(\begin{array}{cc}
d & N t \\
-N & p^{2}
\end{array}\right), \sqrt{-N \tau+p^{2}}\right) \\
& \times \chi_{A}\left(\left(\begin{array}{cc}
1 & -N t \\
N & p^{2} d
\end{array}\right), \sqrt{N \tau+p^{2} d}\right) e\left(b \lambda^{2} / 2\right) \mathfrak{e}_{p \lambda} .
\end{aligned}
$$

Since $d$ is a square modulo $N^{2}$, it is a square modulo the square-free part of $|A|$ and modulo 8. Therefore, a quick calculation shows that the character values are 1 . Consequently,

$$
\rho_{A}\left(L_{\xi}\left(\gamma_{b}\right)\right) \mathfrak{e}_{\lambda}=e\left(b \lambda^{2} / 2\right) \mathfrak{e}_{p \lambda}
$$

In the same way we obtain

$$
\begin{aligned}
\rho_{A}\left(L_{\xi}\left(\beta_{h}\right)\right) \mathfrak{e}_{\lambda} & =\rho_{A}\left(\left(\begin{array}{cc}
r & N h \\
N s & p
\end{array}\right), \sqrt{N s \tau+p}\right) \rho_{A}(\xi) \mathfrak{e}_{\lambda} \\
& =\rho_{A}\left(\left(\begin{array}{cc}
r & N h \\
N s & p
\end{array}\right), \sqrt{N s \tau+p}\right) \mathfrak{e}_{p^{-1} \lambda} \\
& =\chi_{A}\left(\left(\begin{array}{cc}
r & N h \\
N s & p
\end{array}\right), \sqrt{N s \tau+p}\right) \mathfrak{e}_{\lambda} \\
& =\epsilon_{p}^{1-\left(\frac{-1}{|A|}\right)-\operatorname{sig}(A)}\left(\frac{N s}{p}\right)\left(\frac{p}{|A| 2^{\operatorname{sig}(A)}}\right) \mathfrak{e}_{\lambda}
\end{aligned}
$$

Here in the last line we have used the explicit formula for $\chi_{A}$. Since $-N^{2} h s \equiv 1(\bmod p)$, we find

$$
\rho_{A}\left(L_{\xi}\left(\beta_{h}\right)\right) \mathfrak{e}_{\lambda}=\epsilon_{p}^{1-\left(\frac{-1}{|A|}\right)-\operatorname{sig}(A)}\left(\frac{-N h}{p}\right)\left(\frac{p}{|A| 2^{\operatorname{sig}(A)}}\right) \mathfrak{e}_{\lambda}
$$

Now we can compute the Fourier expansion of $\left.f\right|_{k, A} T(\xi)$. We have

$$
\left.f\right|_{k, A} T(\xi)=\left.p^{k-2} f\right|_{k, A} \xi+\left.p^{k-2} \sum_{h(p)^{*}} f\right|_{k, A} L_{\xi}\left(\beta_{h}\right)+\left.p^{k-2} \sum_{b\left(p^{2}\right)} f\right|_{k, A} L_{\xi}\left(\gamma_{b}\right)
$$

For the first summand we find

$$
\begin{aligned}
\left.p^{k-2} f\right|_{k, A} \xi & =p^{k-2} \sum_{\lambda \in A}\left(\left.f_{\lambda}\right|_{k} \alpha\right) \otimes\left(\left.\mathfrak{e}_{\lambda}\right|_{A} \xi\right) \\
& =p^{2 k-2} \sum_{\lambda \in A} f_{\lambda}\left(p^{2} \tau\right) \otimes \mathfrak{e}_{p \lambda} .
\end{aligned}
$$


For the second summand in (4.29) we get

$$
\begin{aligned}
& \left.p^{k-2} \sum_{h(p)^{*}} f\right|_{k, A} L_{\xi}\left(\beta_{h}\right)=p^{k-2} \sum_{h(p)^{*}} \sum_{\lambda \in A}\left(\left.f_{\lambda}\right|_{k} \beta_{h}\right) \otimes\left(\left.\mathfrak{e}_{\lambda}\right|_{A} L_{\xi}\left(\beta_{h}\right)\right) \\
& =\epsilon_{p}^{\operatorname{sig}(A)+\left(\frac{-1}{|A|}\right)-1}\left(\frac{p}{|A| 2^{\operatorname{sig}(A)}}\right) p^{k-2} \sum_{\lambda \in A} \sum_{h(p)^{*}}\left(\frac{-N h}{p}\right) f_{\lambda}(\tau+N h / p) \otimes \mathfrak{e}_{\lambda} .
\end{aligned}
$$

By means of the formula for the Gauss sum $\sum_{h(p)^{*}}\left(\frac{h}{p}\right) e(k h / p)=\left(\frac{k}{p}\right) \epsilon_{p} \sqrt{p}$ we obtain

$$
\sum_{h(p)^{*}}\left(\frac{-N h}{p}\right) f_{\lambda}(\tau+N h / p)=\sqrt{p} \epsilon_{p} \sum_{n \in \mathbb{Z}+\lambda^{2} / 2}\left(\frac{-n}{p}\right) c(\lambda, n) e(n \tau),
$$

and therefore

$$
\begin{aligned}
& \left.p^{k-2} \sum_{h(p)^{*}} f\right|_{k, A} L_{\xi}\left(\beta_{h}\right) \\
& =\epsilon_{p}^{\operatorname{sig}(A)+\left(\frac{-1}{|A|}\right)}\left(\frac{p}{|A| 2^{\operatorname{sig}(A)}}\right) p^{k-3 / 2} \sum_{\lambda \in A} \sum_{n \in \mathbb{Z}+\lambda^{2} / 2}\left(\frac{-n}{p}\right) c(\lambda, n) e(n \tau) \otimes \mathfrak{e}_{\lambda} .
\end{aligned}
$$

Finally, for the third summand in (4.29) we get

$$
\begin{aligned}
\left.p^{k-2} \sum_{b\left(p^{2}\right)} f\right|_{k, A} L_{\xi}\left(\gamma_{b}\right) & =p^{k-2} \sum_{\lambda \in A} \sum_{b\left(p^{2}\right)}\left(\left.f_{\lambda}\right|_{k} \gamma_{b}\right) \otimes\left(\left.\mathfrak{e}_{\lambda}\right|_{A} L_{\xi}\left(\gamma_{b}\right)\right) \\
& =p^{-2} \sum_{\lambda \in A} \sum_{b\left(p^{2}\right)} e\left(-b\left(p^{-1} \lambda\right)^{2} / 2\right) f_{\lambda}\left(\tau / p^{2}+b / p^{2}\right) \otimes \mathfrak{e}_{p^{-1} \lambda} \\
& =\sum_{\lambda \in A} \sum_{n \in \mathbb{Z}+\lambda^{2} / 2} c\left(p \lambda, p^{2} n\right) e(n \tau) \otimes \mathfrak{e}_{\lambda} .
\end{aligned}
$$

This concludes the proof of the theorem.

Remark 4.11. Let $m$ be a positive integer. Let $L$ be the lattice $\mathbb{Z}$ with the quadratic form $x \mapsto-m x^{2}$. Then $L^{\prime}=\frac{1}{2 m} \mathbb{Z}$, and $M_{k, L^{\prime} / L}$ is isomorphic to the space $J_{k+1 / 2, m}$ of Jacobi forms of weight $k+1 / 2$ and index $m$ (cf. [EZ], Theorem 5.1). Under this isomorphism the Hecke operator $T(\xi)$ of Theorem 4.10 corresponds to the Hecke operator $T_{p}$ on $J_{k+1 / 2, m}$ defined in [EZ] $\S 4$ (3). This follows from Theorem 4.10 and [EZ], Theorem 4.5, by comparing the actions on Fourier expansions. Notice that we have in this particular case

$$
\epsilon_{p}^{\operatorname{sig}(A)+\left(\frac{-1}{|A|}\right)}\left(\frac{p}{|A| 2^{\operatorname{sig}(A)}}\right)=\left(\frac{m}{p}\right) .
$$

For the lattice $L=\mathbb{Z}$ with the quadratic form $x \mapsto m x^{2}$ the space $M_{k, L^{\prime} / L}$ is isomorphic to the space $J_{k+1 / 2, m}^{\text {skew }}$ of skew holomorphic Jacobi forms of weight $k+1 / 2$ and index $m$ defined in $[\mathrm{Sk}]$. Again, the Hecke operators of Theorem 4.10 correspond to the usual Hecke operators on skew-holomorphic Jacobi forms. 
4.3. A Hecke algebra on vector valued modular forms. Using the double coset actions of the previous section, we may define for every positive integer $m$ coprime to $N$ a Hecke operator $T\left(m^{2}\right)^{*}: M_{k, A} \rightarrow M_{k, A}$, by

$$
\left.f \mapsto f\right|_{k, A} T\left(m^{2}\right)^{*}= \begin{cases}\left.f\right|_{k, A} T\left(\left(\begin{array}{cc}
m^{2} & 0 \\
0 & 1
\end{array}\right), m\right), & \text { if } \operatorname{sig}(A) \text { is even, } \\
\left.f\right|_{k, A} T\left(\left(\begin{array}{cc}
m^{2} & 0 \\
0 & 1
\end{array}\right), 1, m, 1\right), & \text { if } \operatorname{sig}(A) \text { is odd. }\end{cases}
$$

In the case of even signature (that is integral weight), the operator $T\left(m^{2}\right)^{*}$ differs from the usual Hecke operator $T\left(m^{2}\right)$ which is given by the sum of double cosets consisting of all integral matrices of determinant $m^{2}$. This is the reason for our notation. In the case of odd signature (that is half-integral weight), the operator $T\left(m^{2}\right)^{*}$ is analogous to the Hecke operator in Sh2] on scalar valued modular forms.

Theorem 4.12. The Hecke operators $T\left(m^{2}\right)^{*}$ (for $m$ coprime to $N$ ) generate a commutative subalgebra of $\operatorname{End}\left(M_{k, A}\right)$, which is actually already generated by the $T\left(p^{2}\right)^{*}$ for $p$ prime and coprime to $N$. The operators $T\left(m^{2}\right)^{*}$ take cusp forms to cusp forms and are self-adjoint with respect to the Petersson scalar product.

Proof. Using the actions (4.5) and (4.20), this follows in the usual way from the properties of the abstract Hecke algebra of the pair $(\mathcal{Q}(N), \Gamma)$, respectively $\left(\mathcal{Q}_{2}(N), \Delta\right)$.

\section{The Weil Representation on double Cosets}

We now want to define Hecke operators $T\left(m^{2}\right)^{*}$ as in Section 4.3 for all positive integers $m$, not necessarily coprime to $N$. If $m$ and $N$ are not coprime, then the reduction of $\left(\begin{array}{cc}m^{2} & 0 \\ 0 & 1\end{array}\right)$ does not belong to $\mathrm{GL}_{2}(\mathbb{Z} / N \mathbb{Z})$. So we cannot use the results of the previous sections. However, it is still possible to extend the Weil representation $\rho_{A}$ to the corresponding double coset in a compatible way, as we will see.

Let $m$ be a positive integer and $\alpha=\left(\left(\begin{array}{cc}m^{2} & 0 \\ 0 & 1\end{array}\right), 1\right) \in \widetilde{G L}_{2}^{+}(\mathbb{R})$. We define a right action on $\mathbb{C}[A]$ by

$$
\left.\mathfrak{e}_{\lambda}\right|_{A} \alpha=\mathfrak{e}_{m \lambda} .
$$

To lighten the notation, we will frequenty drop the subscript from the slash operator. Comparing with (3.6) and (4.13), we see that (5.1) is compatible with our earlier definition in the case that $(m, N)=1$. Moreover, if $\delta=\gamma \alpha \gamma^{\prime} \in \tilde{\Gamma} \alpha \tilde{\Gamma}$, we put

$$
\mathfrak{e}_{\lambda}\left|\delta=\mathfrak{e}_{\lambda}\right| \gamma|\alpha| \gamma^{\prime}
$$

We now show that this right action is well defined, that is, independent of the decomposition of $\delta$.

Proposition 5.1. Let $\delta=\gamma \alpha \gamma^{\prime}=\gamma_{1} \alpha \gamma_{1}^{\prime} \in \tilde{\Gamma} \alpha \tilde{\Gamma}$ (where $\left.\gamma, \gamma^{\prime}, \gamma_{1}, \gamma_{1}^{\prime} \in \tilde{\Gamma}\right)$. Then

$$
\mathfrak{e}_{\lambda}|\gamma| \alpha\left|\gamma^{\prime}=\mathfrak{e}_{\lambda}\right| \gamma_{1}|\alpha| \gamma_{1}^{\prime}
$$

Proof. First, one easily shows that it suffices to prove the proposition in the case that $\gamma^{\prime}=\gamma_{1}=1$. So we have $\delta=\gamma \alpha=\alpha \gamma_{1}^{\prime}$ and need to show that

$$
\mathfrak{e}_{\lambda}|\gamma| \alpha=\mathfrak{e}_{\lambda}|\alpha| \gamma_{1}^{\prime} \text {. }
$$


If we write $\gamma=\left(\left(\begin{array}{ll}a & b \\ c & d\end{array}\right), \pm \sqrt{c \tau+d}\right)$, then $\gamma_{1}^{\prime}=\left(\left(\begin{array}{cc}a & b / m^{2} \\ m^{2} c & d\end{array}\right), \pm \sqrt{m^{2} c \tau+d}\right)$. In particular, $\gamma \in \tilde{\Gamma}^{0}\left(m^{2}\right)$ and $\gamma_{1}^{\prime} \in \tilde{\Gamma}_{0}\left(m^{2}\right)$. It suffices to prove (5.3) for $\gamma$ in a set of generators of $\tilde{\Gamma}^{0}\left(m^{2}\right)$.

It is easily seen that $\tilde{\Gamma}^{0}\left(m^{2}\right)$ is generated by $\tilde{\Gamma}^{0}\left(m^{2}\right) \cap \tilde{\Gamma}_{0}^{0}(N), T^{m^{2}}$, and $U$. For $\gamma \in$ $\tilde{\Gamma}^{0}\left(m^{2}\right) \cap \tilde{\Gamma}_{0}^{0}(N)$ the identity (5.3) immediately follows from Proposition 2.2. For $\gamma=T^{m^{2}}$ it is easily verified as well.

We now consider (5.3) for $\gamma=U$. Using Lemma 2.3, we see that the left hand side of (5.3) is equal to

$$
\mathfrak{e}_{\lambda}|U| \alpha=\frac{1}{|A|} \sum_{\mu, \nu \in A} e\left(\mu^{2} / 2+(\mu, \lambda-\nu)\right) \mathfrak{e}_{m \nu} .
$$

Using (2.2), in the sum over $\nu$ we write $\nu=\nu^{\prime} / m+\nu^{\prime \prime}$ where $\nu^{\prime} \in A^{m}$ and $\nu^{\prime \prime} \in A_{m}$. We obtain

$$
\mathfrak{e}_{\lambda}|U| \alpha=\frac{1}{|A|} \sum_{\mu \in A} \sum_{\substack{\nu^{\prime} \in A^{m} \\ \nu^{\prime \prime} \in A_{m}}} e\left(\mu^{2} / 2+\left(\mu, \lambda-\nu^{\prime} / m-\nu^{\prime \prime}\right)\right) \mathfrak{e}_{\nu^{\prime}}
$$

The sum over $\nu^{\prime \prime}$ is equal to $\left|A_{m}\right|$ if $\mu \in A^{m}$ and 0 otherwise. Hence

$$
\begin{aligned}
\mathfrak{e}_{\lambda}|U| \alpha & =\frac{\left|A_{m}\right|}{|A|} \sum_{\mu \in A^{m}} \sum_{\nu \in A^{m}} e\left(\mu^{2} / 2+(\mu, \lambda-\nu / m)\right) \mathfrak{e}_{\nu} \\
& =\frac{1}{|A|} \sum_{\mu \in A} \sum_{\nu \in A^{m}} e\left((m \mu)^{2} / 2+(m \mu, \lambda-\nu / m)\right) \mathfrak{e}_{\nu} .
\end{aligned}
$$

On the other hand, the right hand side of (5.3) is equal to

$$
\begin{aligned}
\mathfrak{e}_{\lambda}|\alpha| U^{m^{2}} & =\frac{1}{|A|} \sum_{\mu, \nu \in A} e\left(m^{2} \mu^{2} / 2+(\mu, m \lambda-\nu)\right) \mathfrak{e}_{\nu} \\
& =\frac{1}{|A|\left|A_{m}\right|} \sum_{\mu, \nu \in A} \sum_{\mu^{\prime} \in A_{m}} e\left(m^{2} \mu^{2} / 2+\left(\mu+\mu^{\prime}, m \lambda-\nu\right)\right) \mathfrak{e}_{\nu} .
\end{aligned}
$$

The sum over $\mu^{\prime}$ is equal to $\left|A_{m}\right|$ if $\nu \in A^{m}$ and 0 otherwise. Hence

$$
\begin{aligned}
\mathfrak{e}_{\lambda}|\alpha| U^{m^{2}} & =\frac{1}{|A|} \sum_{\mu \in A} \sum_{\nu \in A^{m}} e\left(m^{2} \mu^{2} / 2+(\mu, m \lambda-\nu)\right) \mathfrak{e}_{\nu} \\
& =\frac{1}{|A|} \sum_{\mu \in A} \sum_{\nu \in A^{m}} e\left((m \mu)^{2} / 2+(m \mu, \lambda-\nu / m)\right) \mathfrak{e}_{\nu} .
\end{aligned}
$$

This concludes the proof of the proposition.

Lemma 5.2. Let $\delta \in \tilde{\Gamma} \alpha \tilde{\Gamma}$, and let $\gamma, \gamma^{\prime} \in \tilde{\Gamma}$. Then

$$
\mathfrak{e}_{\lambda}\left|\left(\gamma \delta \gamma^{\prime}\right)=\mathfrak{e}_{\lambda}\right| \gamma|\delta| \gamma^{\prime}
$$

Proof. This follows immediately from the definition and Proposition 5.1. 
The element $\beta=\left(\left(\begin{array}{cc}1 & 0 \\ 0 & m^{2}\end{array}\right), m\right) \in \widetilde{\mathrm{GL}}_{2}^{+}(\mathbb{R})$ belongs to the double coset $\tilde{\Gamma} \alpha \tilde{\Gamma}$. The following Proposition gives its action on $\mathbb{C}[A]$.

Proposition 5.3. We have

$$
\mathfrak{e}_{\lambda} \mid \beta=\sum_{\substack{\mu \in A \\ m \mu=\lambda}} \mathfrak{e}_{\mu} .
$$

Moreover, for the standard scalar product on $\mathbb{C}[A]$ we have

$$
\langle\mathfrak{a} \mid \alpha, \mathfrak{b}\rangle=\langle\mathfrak{a}, \mathfrak{b} \mid \beta\rangle, \quad \mathfrak{a}, \mathfrak{b} \in \mathbb{C}[A] .
$$

Proof. The first assertion follows from the fact that $\beta=S \alpha S^{-1}$ and Lemma 5.2. The second assertion is verified by a straightforward computation.

Proposition 5.4. Let $m, n$ be coprime positive integers, and put $\alpha=\left(\left(\begin{array}{cc}m^{2} & 0 \\ 0 & 1\end{array}\right), 1\right)$ and $\beta=\left(\left(\begin{array}{cc}n^{2} & 0 \\ 0 & 1\end{array}\right), 1\right)$. Then for $g \in \tilde{\Gamma} \alpha \tilde{\Gamma}$ and $h \in \tilde{\Gamma} \beta \tilde{\Gamma}$ we have

$$
\mathfrak{e}_{\lambda}|g| h=\mathfrak{e}_{\lambda} \mid(g h) .
$$

Proof. We write $g=\gamma \alpha \gamma^{\prime}$ and $h=\delta \beta \delta^{\prime}$ with $\gamma, \gamma^{\prime}, \delta, \delta^{\prime} \in \tilde{\Gamma}$. Since $(m, n)=1$, a simple argument using the elementary divisor theorem shows that $g h=\epsilon \alpha \beta \epsilon^{\prime}$ for suitable $\epsilon, \epsilon^{\prime} \in \tilde{\Gamma}$.

In view of Lemma 5.2 it suffices to prove the assertion in the case that $\gamma=\delta^{\prime}=1$.

As a second reduction step, we now show that we may in addition assume that $\epsilon^{\prime}=1$. In fact, the identity $g h=\alpha \gamma^{\prime} \delta \beta=\epsilon \alpha \beta \epsilon^{\prime}$ implies that

$$
\delta \beta \epsilon^{\prime-1}=\gamma^{\prime-1} \alpha^{-1} \epsilon \alpha \beta .
$$

The matrix component of the left hand side has integral entries, hence the same is true for the right had side. Using the coprimality of $m$ and $n$ we may infer that

$$
\tilde{\delta}:=\gamma^{\prime-1} \alpha^{-1} \epsilon \alpha
$$

belongs to $\tilde{\Gamma}$. We obtain

$$
\begin{aligned}
\delta \beta & =\tilde{\delta} \beta \epsilon^{\prime}, \\
\left(\alpha \gamma^{\prime}\right)(\tilde{\delta} \beta) & =\epsilon \alpha \beta .
\end{aligned}
$$

From the assertion in the $\epsilon^{\prime}=1$ case we get

$$
\mathfrak{e}_{\lambda}\left|\left(\alpha \gamma^{\prime}\right)\right|(\tilde{\delta} \beta)=\mathfrak{e}_{\lambda} \mid(\epsilon \alpha \beta),
$$

which implies the assertion for arbitrary $\epsilon^{\prime}$.

Finally we need to prove the claim in the case that $\gamma=\delta^{\prime}=\epsilon^{\prime}=1$. So $g=\alpha \gamma^{\prime}, h=\delta \beta$ and

$$
g h=\alpha \gamma^{\prime} \delta \beta=\epsilon \alpha \beta .
$$

Since $\alpha \gamma^{\prime} \delta=\epsilon \alpha$, Lemma 5.2 implies that

$$
\begin{aligned}
\mathfrak{e}_{\lambda} \mid\left(\alpha \gamma^{\prime} \delta\right) & =\mathfrak{e}_{\lambda} \mid(\epsilon \alpha), \\
\mathfrak{e}_{\lambda}\left|\left(\alpha \gamma^{\prime} \delta\right)\right| \beta & =\mathfrak{e}_{\lambda}|(\epsilon \alpha)| \beta .
\end{aligned}
$$

Now the claim follows from Lemma 5.2 and the fact that $\mathfrak{e}_{\lambda}|\alpha| \beta=\mathfrak{e}_{\lambda} \mid(\alpha \beta)$. 
Definition 5.5. Let $m$ be a positive integer and $\alpha=\left(\left(\begin{array}{cc}m^{2} & 0 \\ 0 & 1\end{array}\right), 1\right) \in \widetilde{\mathrm{GL}}_{2}^{+}(\mathbb{R})$. Let

$$
\tilde{\Gamma} \cdot \alpha \cdot \tilde{\Gamma}=\bigcup_{i} \tilde{\Gamma} \cdot \delta_{i}
$$

be a disjoint left coset decomposition. We define the Hecke operator $T\left(m^{2}\right)^{*}$ on modular forms $f \in M_{k, A}$ by

$$
\left.f \mapsto f\right|_{k, A} T\left(m^{2}\right)^{*}=\left.m^{k-2} \sum_{i} f\right|_{k, A} \delta_{i}=m^{k-2} \sum_{i} \sum_{\lambda \in A}\left(\left.f_{\lambda}\right|_{k} \delta_{i}\right) \otimes\left(\left.\mathfrak{e}_{\lambda}\right|_{A} \delta_{i}\right) .
$$

Lemma 5.2 implies that the definition does not depend on the choice of the coset representatives. Notice that for $m$ coprime to $N$, this definition agrees with the earlier definition in Section 4.3 .

Theorem 5.6. For any positive integer $m$, the Hecke operator $T\left(m^{2}\right)^{*}$ is a linear operator on $M_{k, A}$ taking cusp forms to cusp forms. It is self adjoint with respect to the Petersson scalar product. Moreover, if $m, n$ are coprime, then

$$
T\left(m^{2}\right)^{*} T\left(n^{2}\right)^{*}=T\left(m^{2} n^{2}\right)^{*} .
$$

Proof. The first assertion is a consequence of Lemma 5.2. The self adjointness follows from Proposition 5.3 along the standard argument for scalar valued modular forms (cf. [Bu], Theorem 1.4.3). The last assertion is a consequence of Proposition 5.4 and the corresponding property of the abstract Hecke algebra.

Remark 5.7. For a prime $p$ dividing $N$ the local Hecke algebra, that is, the subalgebra of $\operatorname{End}\left(M_{k, A}\right)$ generated by the $T\left(p^{2 \nu}\right)^{*}$, is considerably more complicated than in the case where $p$ is coprime to $N$. For instance, it is commutative if $p$ is coprime to $N$, but in general non-commutative if $p$ divides $N$.

\section{REFERENCES}

[Bö] S. Böcherer, Über die Funktionalgleichung automorpher L-Funktionen zur Siegelschen Modulgruppe, J. Reine Angew. Math. 362 (1985), 146-168.

[Bo1] R. Borcherds, Automorphic forms with singularities on Grassmannians, Inv. Math. 132 (1998), 491-562.

[Bo2] R. Borcherds, Reflection groups of Lorentian lattices, Duke Math. J. 104 (2000), 319-366.

[Br] J. H. Bruinier, Borcherds products on $\mathrm{O}(2, l)$ and Chern classes of Heegner divisors, Springer Lecture Notes in Mathematics 1780, Springer-Verlag (2002).

[BB] J. H. Bruinier and M. Bundschuh, On Borcherds products associated with lattices of prime discriminant, Ramanujan J. 7 (2003), 49-61.

[Bu] D. Bump, Automorphic Forms and Representations, Cambridge University Press (1998).

[CS] J. H. Conway and H. J. Sloane, Sphere packings, lattices and groups. Third edition. Grundlehren der Mathematischen Wissenschaften, 290, Springer-Verlag, New York (1999).

[Eb] W. Ebeling, Lattices and codes. A course partially based on lectures by F. Hirzebruch. Second revised edition. Advanced Lectures in Mathematics, Vieweg, Braunschweig (2002).

[EZ] M. Eichler and D. Zagier, The Theory of Jacobi Forms, Progress in Math. 55, Birkhäuser (1985).

$[\mathrm{FH}] \quad$ W. Fulton and J. Harris, Representation Theory, Springer GTM 129, Springer Verlag (1991). 
[Ga] P. Garrett, Pullbacks of Eisenstein series; Applications. In: Automorphic forms of several variables, Taniguchi Symposium, Katata, 1983, Birhäuser (1984).

$[\mathrm{McG}]$ W. J. McGraw, The rationality of vector valued modular forms associated with the Weil representation, Math. Ann. 326 (2003), 105-122.

$[\mathrm{MH}] \quad$ J. Milnor and D. Husemoller, Symmetric Bilinear Forms, Ergebnisse der Mathematik und ihrer Grenzgebiete 73, Springer-Verlag, New York-Heidelberg (1973).

[Mi] T. Miyake, Modular forms, Springer, 1989.

[Ni] V. V. Nikulin, Integer symmetric bilinear forms and some of their geometric applications. (Russian) Izv. Akad. Nauk SSSR Ser. Mat. 43 (1979), 111-177, 238. English translation in Mathematics of the U.S.S.R., Izvestia 14 (1980), 103-167.

$[\mathrm{NW}]$ A. Nobs and J. Wolfart, Die irreduziblen Darstellungen der Gruppen $\mathrm{SL}_{2}\left(\mathbb{Z}_{p}\right)$, insbesondere $\mathrm{SL}_{2}\left(\mathbb{Z}_{2}\right)$. I. Teil, Comment Math. Helvetici 51 (1976), 491-526.

[PSR] I. Piatetski-Shapiro, S. Rallis, L-functions for classical groups, Lecture Notes in Mathematics 1254, Springer-Verlag, Berlin (1987).

[Sch] N. R. Scheithauer, On the classification of automorphic products and generalized Kac-Moody algebras, Invent. Math. 164 (2006), 641-678.

[Sh1] G. Shimura, Introduction to the Arithmetic Theory of Automorphic Functions, Princeton University Press, Princeton (1971).

[Sh2] G. Shimura, On modular forms of half integral weight, Annals of Math. 97 (1973), 440-481.

[Shin] T. Shintani, On the construction of holomorphic cusp forms of half integral weight, Nagoya Math. J. 58 (1975), 83-126.

[Sk] N.-P. Skoruppa, Developments in the theory of Jacobi forms. In: Proceedings of the conference on automorphic funtions and their applications, Chabarovsk (eds.: N. Kuznetsov and V. Bykovsky), The USSR Academy of Science (1990), 167-185. (see also MPI-preprint 89-40, Bonn (1989).)

Mathematisches Institut, Universität ZU KÖLn, Weyertal 86-90, D-50931 Köln, Germany E-mail address: bruinier@math.uni-koeln.de

E-mail address: ostein@math.uni-koeln.de 